\title{
A case of imported malaria: the first report of a Plasmodium malariae infection in Sri Lanka after 37 years
}

\author{
H A C Hapuarachchi ${ }^{1}$, N K Gunawardane ${ }^{1}$, M P Senevirathne ${ }^{2}$, W Abeyewickreme ${ }^{1}$ and N R de Silva ${ }^{1}$ \\ (Index words: Plasmodium malariae, Plasmodium falciparum, PCR, microscopy)
}

\section{Summary}

We report a case of Plasmodium falciparum and $P$ malariae mixed infection in a patient who had been living in Malawi. This is the first case of $P$ malariae reported in Sri Lanka in 4 decades. The presence of both parasites was confirmed by microscopy and polymerase chain reaction (PCR). The history strongly indicated that the infection had been acquired from Malawi. The patient had liver dysfunction and a transient glomerulonephritis, both of which subsided with antimalarial treatment.

\section{Introduction}

We report a case of imported malaria in a patient who returned from Malawi with a mixed infection of Plasmodium falciparum and $P$ malariae. $P$ malariae infection has not been reported in Sri Lanka since 1969 [1].

\section{Case report}

A 51-year old man was admitted to the North Colombo Teaching Hospital with high intermittant fever with chills and rigors for 2 weeks, despite treatment by a general practitioner. Fever was accompanied by severe headache, myalgia, arthralgia, vomiting, loss of appetite and backache. The patient had developed fever 3 days after his return from Malawi where he had been working as an engineer for 8 years. He was febrile and icteric, but not anaemic. There was mild hepatomegaly without splenomegaly. The serum bilirubin was $4.8 \mathrm{mg} / \mathrm{dl}$ and serum liver enzymes were raised (SGOT $>125 \mathrm{U} / 1$, SGPT $>250 \mathrm{U} / \mathrm{l}$ ). His haemoglobin level was $12.8 \mathrm{~g} / \mathrm{dl}$, the total white blood cell count was $10400 / \mu$ l and platelet count was $230000 / \mu 1$. Urine analysis showed proteinuria (albumin ++ ), bile (+) and red blood cells (RBC) (4-6 per field). However, his blood urea and urine output were normal.

Microscopical examination of Giemsa stained thin and thick blood smears showed an asexual malaria parasite density of 120000 per $\mu$ l of blood. Infected RBCs were not enlarged. The presence of double-chromatin and applique form trophozoites, with occasional multiple invasion suggested $P$ falciparum infection. In addition, there were characteristic "band forms" trophozoites of $P$ malariae (figure 1) [2]. Presence of both $P$ falciparum [3] and $P$ malariae [4] was confirmed by polymerase chain reaction (PCR) as described previously. Because of the high $P$ falciparum burden, the patient was treated with parenteral quinine and primaquine according to the national Anti-Malaria Campaign (AMC) guidelines. Parasitaemia disappeared within 3 days of treatment, and there was no recrudescence during the 28 days follow up period.

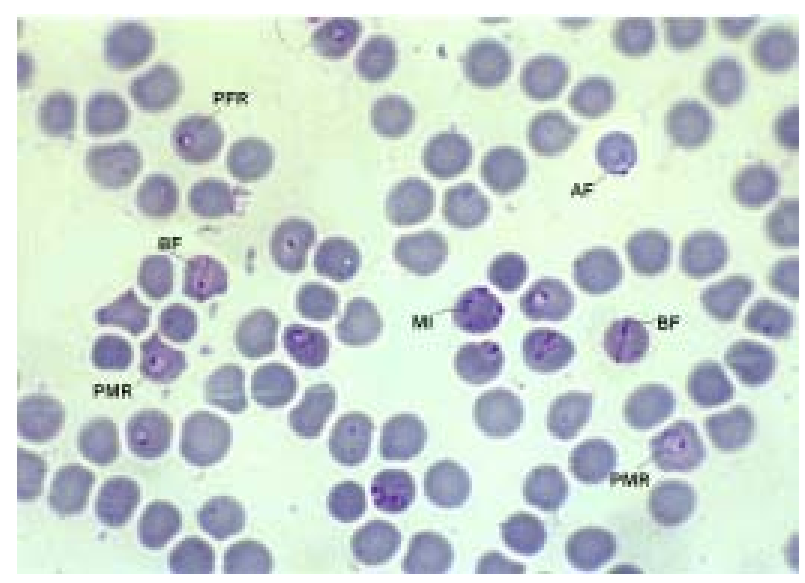

Figure 1. Asexual stages of $\boldsymbol{P}$ malariae and $\boldsymbol{P}$ falciparum seen in the thin blood smear. This picture shows different types of $\boldsymbol{P}$ falciparum ring trophozoite stages, including the applique form. This also shows several RBCs with multiple infections. The infected RBCs are not enlarged. In addition, characteristic "bandform" and ring trophozoites of $\boldsymbol{P}$ malariae can also be seen. AF - applique form, BF - band form of $P$ malariae, MI-RBC with a multiple infection, PFR - $P$ falciparum ring trophozoite, PMR - $P$ malariae ring trophozoite, Giemsa, x 100.

\section{Discussion}

In malaria, increased demand for hepatic activity to clear infected RBCs results in liver hyperplasia and hepatocellular damage [5]. This explains the presence of hepatomegaly and impaired liver functions in this patient.

${ }^{1}$ Department of Parasitology, Faculty of Medicine, P.O. Box 6, Thalagolla Road, Ragama, Sri Lanka. ${ }^{2}$ Colombo North Teaching Hospital, Ragama, Sri Lanka.

Correspondence: $\mathrm{HACH}$, e-mail <chandith@lycos.com>. Received 31 October 2007 and revised version accepted 28 February 2008. Competing interests: none declared. 
He also had evidence of renal dysfunction, which improved dramatically with antimalarial therapy. Renal pathology in malaria clinically manifests either as glomerulonephritis or acute renal failure. The presence of self-limiting proteinuria and microscopic haematuria with normal renal function was suggestive of a transient glomerulonephritis caused by $P$ falciparum rather than a chronic progressive glomerulonephritis (malarial nephropathy) seen in P malariae infections [5]. However, the presence of $P$ malariae necessitates long-term follow up of this patient, due to its well known ability to cause chronic infection.

One of the main disadvantages of microscopy as well as the available rapid diagnostic kits for malaria is poor sensitivity in detecting mixed species infections. In this particular case, even though microscopy was suggestive of $P$ malariae infection, a more precise identification was essential due to its absence in Sri Lanka for nearly 4 decades [1]. The most appropriate diagnostic option available was PCR, which has high sensitivity and species specificity [6]. Because of the high dependence on light microscopy for malaria diagnosis, it is possible that similar cases may have been misdiagnosed previously among immigrants to Sri Lanka. Many mosquito vectors found in Sri Lanka, including Anopheles culicifacies, are capable of transmitting all human malaria species, and misdiagnosis increases the risk of establishing new species in the country. Though the incidence of malaria has fallen in the country in recent years [7], this case report emphasizes the need of physicians to be vigilant about the types of malaria in immigrants, especially those from Africa and southeast Asia. It also highlights the importance of proper screening for infectious diseases among visitors to the country from high risk regions.

\section{Acknowledgements}

We thank the medical and nursing staff of ward No. 9 of Colombo North Teaching Hospital, Ragama, Mr. S. Abeysundara of the Department of Parasitology, Faculty of Medicine, Ragama, Staff of the Molecular Medicine Unit, Faculty of Medicine, Ragama and Drs. S. Leeminsawat and P. Chavalitshewinkoon-petmitr of the Department of Medical Entomology, Faculty of Tropical Medicine, Mahidol University, Thailand for their assistance in the diagnosis, management and follow-up of this patient.

\section{References}

1. Edirisinghe JS. Historical references to malaria in Sri Lanka and some notable episodes up to present times. Ceylon Medical Journal 1988; 33: 110-7.

2. Garnham PCC. Malaria parasites of man: life cycles and morphology. In: Wernsdorfer WH, McGregor I eds. Malaria: Principals and Practice of Malariology. London: Churchill Livingston, 1988: 61-96.

3. Viriyakosol S, Siripoon N, Petcharapirat C, Petcharapirat P, Jarra W, Thaithong S, Brown KN, Snounou G. Genotyping of Plasmodium falciparum isolates by the polymerase chain reaction and potential uses in epidemiological studies. Bulletin of the World Health Organization 1995; 73: 85-95.

4. Rosenblatt J, Sloan L, Swan H, ChavalitshewinkoonPetmitr P, Looareesuwan S, Muyombwe A, Krudsood S, Leowattana W, Wilairatana P. Evaluation of a real-time PCR assay for the diagnosis of malaria in patients from Thailand. American Journal for Tropical Medicine and Hygiene 2005; 73: 850-4.

5. Boonpucknavig V, Boonpucknavig S. The histopathology of malaria. In: Wernsdorfer WH, McGregor I eds. Malaria: Principals and Practice of Malariology. London: Churchill Livingston, 1988: 673-708.

6. Snounou G, Viriyakosol S, Jarra W, Thaithong S, Brown $\mathrm{KN}$. Identification of the four human malaria parasite species in field samples by the polymerase chain reaction and detection of a high prevalence of mixed infections. Molecular and Biochemical Parasitology 1993; 58: 283-92.

7. Ministry of Health. Annual Health Bulletin. Colombo, Sri Lanka 2005. 